\title{
BMJ Open Impact of Ramadan fasting on kidney function and related outcomes in patients with chronic kidney disease: a systematic review protocol
}

Aminu K Bello, Julia Kurzawa, Mohamed A Osman, Michelle E Olah, Anita Lloyd, Natasha Wiebe, Syed Habib, Uwais Qarni, Soroush Shojai, Robert P Pauly

To cite: Bello AK, Kurzawa J, Osman MA, et al. Impact of Ramadan fasting on kidney function and related outcomes in patients with chronic kidney disease: a systematic review protocol. BMJ Open 2019;9:e022710. doi:10.1136/ bmjopen-2018-022710

- Prepublication history and additional material for this paper are available online. To view please visit the journal (http:// dx.doi.org/10.1136/bmjopen2018-022710).

Received 22 March 2018 Revised 11 December 2018 Accepted 19 December 2018

A Check for updates

(C) Author(s) (or their employer(s)) 2019. Re-use permitted under CC BY-NC. No commercial re-use. See rights and permissions. Published by BMJ.

Department of Medicine, University of Alberta, Edmonton, Alberta, Canada

Correspondence to

Dr Robert P Pauly;

rpauly@ualberta.ca

\section{ABSTRACT}

Introduction Fasting during the month of Ramadan is a significant Islamic religious practice that involves abstinence from food, drink and medication from dawn to dusk. As just under a quarter of the world's population identifies as Muslim, the effect of fasting on chronic conditions, such as chronic kidney disease (CKD) is a topic of broad relevance. To date, the information in this area has been mixed, with many limitations of previous studies. This study aims to synthesise the evidence of the effect of Ramadan fasting on changes on kidney function, risk factors, episodes of acute kidney injury and impact on the quality of life in patients with CKD or kidney transplant. Methods and analysis A systematic review of the literature will be conducted, using electronic databases such as MEDLINE, Embase, Global Health, CINAHL and Scopus. Original research and grey literature on the effect of Ramadan fasting in adult patients with CKD or renal transplantation will be included. Two reviewers will independently screen articles for inclusion in the review and independently assess the methodology of included studies using a customised checklist. Mean difference or risk ratio will be reported for continuous or dichotomous outcomes and results will be pooled using a randomeffects model where heterogeneity is reasonable. If possible, subgroups (CKD status, setting, season and risk of bias) will be analysed for effect modification with fasting and the outcomes of interest. Risk of bias will be assessed using the Downs and Black checklist.

Ethics and dissemination The results will be disseminated using a multifaceted approach to engage all stakeholders (patients, practitioners and community leaders). Research ethics board approval is not required as this is a systematic review of previously published research.

PROSPERO registration number CRD42018088973.

\section{INTRODUCTION}

Ramadan represents the holiest month of the year for Muslims. Fasting during Ramadan is a significant religious practice and a key pillar of Islam. Ramadan fasting involves strict abstinence from food, drink and medication from sunrise to sunset. On average, this period of

\section{Strengths and limitations of this study}

- To our knowledge, this review is the first to leverage a rigorous methodology to review evidence of the effect of Ramadan fasting on a wide range of adverse renal health outcomes.

- Because of the variations in fasting hours and climate zones, subgroup analyses will be conducted, where it is possible, for chronic kidney disease stage, latitude and season.

- Anticipated limitations include: heterogeneity of included studies including design, setting (geography) and population.

fasting lasts between 12 and 14 hours, however, it may extend up to 22 hours depending on the season and latitude (figure 1) (online supplementary table S1). ${ }^{1-3}$ As Ramadan follows the lunar calendar year (Hijri), it may occur in any season (box 1).

Globally, approximately $23 \%$ of individuals identify as Muslim. ${ }^{4}$ Within Canada, Islam represents the second largest and fastest-growing religion. ${ }^{5} 6$ In an increasingly globalised and interconnected world, physicians practising in all countries are confronted with questions from patients with chronic conditions about the safety of Ramadan fasting. Travellers, children, women who are menstruating, pregnant or breastfeeding, and anyone with illness are exempt from fasting. Despite this exemption, many still wish to participate in the fast, including patients with chronic kidney disease (CKD) and renal transplant recipients. ${ }^{7}$

Ramadan fasting can have positive spiritual, social and psychological effects. ${ }^{8} 9$ However, there are conflicting data regarding various physiological outcomes including renal function. Some studies have reported a temporary improvement in estimated glomerular filtration rate (eGFR) and reduced proteinuria in 


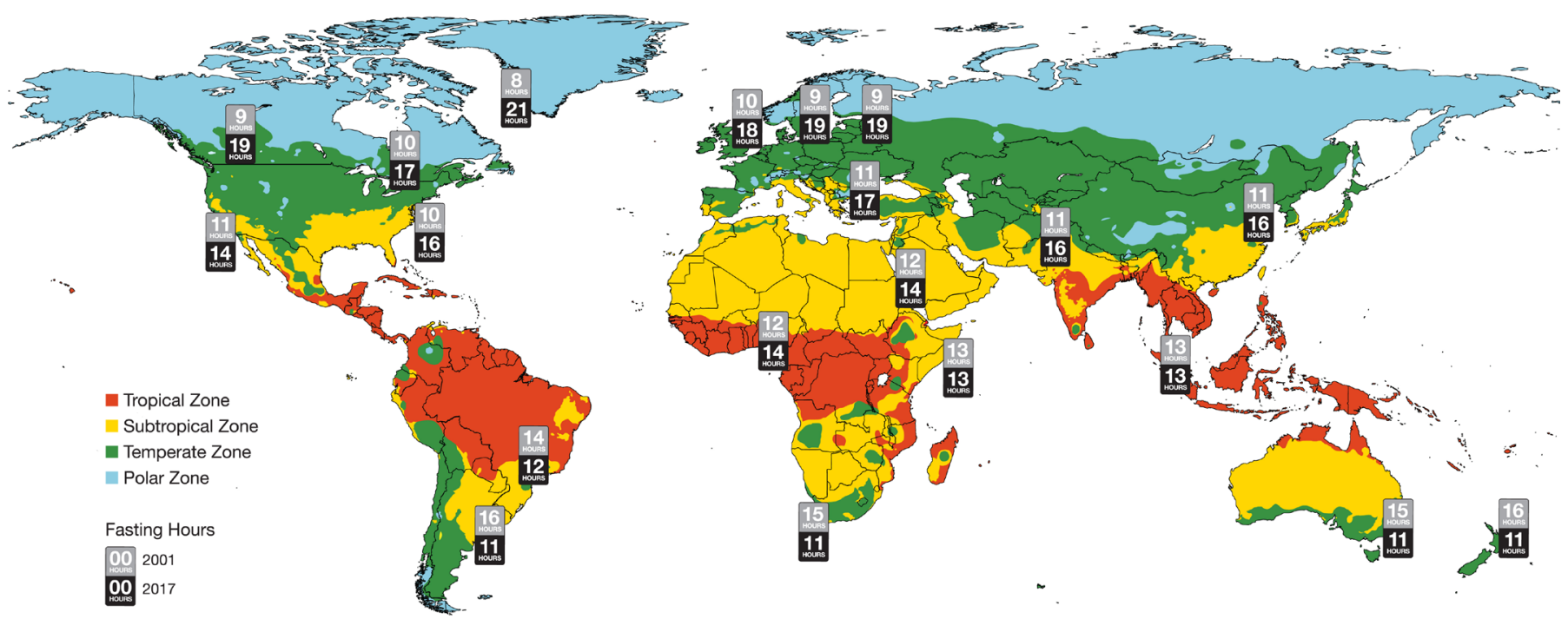

Figure 1 World map depicting four major climate zones and average fasting hours in selected countries in Ramadan 2017/2001. World map colour-coded based on four different climate zones adopted and modified from the Koppen climate $\mathrm{map}^{3}$ and modified into the SpinCushion map. The hours are an estimate of average fasting hours of selected world countries during Ramadan in the years 2017 (black box) and 2001 (grey box). Description of the four climate zones (plant-based), average temperature or precipitation (criteria based) and day length variations across the year: tropical zone (type A): average temperatures of $\geq 18^{\circ} \mathrm{C}$, equal day length most of the year; subtropical zone (type B): deficient precipitation during most of the year, mild day length variation; temperate zone (type C/D): at least 1 month averaging below $0^{\circ} \mathrm{C}$, significant day length variation; polar zone (type E): every month of the year with an average temperature below $10^{\circ} \mathrm{C}$, largest day length variation. Note: Ramadan fasting hours are not fixed as the fasting period follows the lunar calendar (the period generally moves 10 to 11 days back each year). The time change mostly affects fasting hours in countries located in the polar and temperate zones with minimal effects on fasting hours in countries situated in tropical and subtropical areas. For instance, in Edmonton, Canada, the average fasting hours during Ramadan in 2017 (May/Jun) was 19 hours, whereas the average fasting hours, during Ramadan 2001 (Nov/Dec) was 9 hours - a change of 10 hours. In Mecca, Saudi Arabia the average fasting hours during Ramadan in 2017 (May/Jun) was 14 hours, whereas the average fasting hours, during Ramadan 2001 (Nov/Dec) was 12 hours - a change of 2 hours (online supplementary table S1).

patients with CKD or kidney transplantation, ${ }^{10}$ whereas other studies demonstrated a negative effect on kidney and cardiovascular health outcomes. ${ }^{11-13}$

This primary aim of the study is to conduct a systematic review that summarises all available literature on the

\section{Box 1 Summary points}

- Ramadan is the ninth lunar month of the Islamic calendar.

- Fasting during Ramadan is one of the five pillars of Islam.

- Fasting involves abstaining from food, drink, medication and sexual intercourse during daylight.

- As part of other exemptions, patients (both acute and chronic) are exempt from fasting during Ramadan.

- As Ramadan follows the lunar calendar, it can occur in any season of the year, and fasting hours on average are 12 to 14 hours (but can last up to 22 hours).

- The effects of long daily fasting hours (especially at extreme northern and southern latitudes during their respective summers) on kidney function in patients with CKD are not known.

CKD, chronic kidney disease. effect of Ramadan fasting on kidney function in patients living with CKD or kidney transplantation. We will also examine the associations of fasting with changes in CKD risk factors (ie, blood pressure, blood glucose, proteinuria and body mass index), episodes of acute kidney injury (AKI), adverse health outcomes (all-cause hospitalisation, cardiovascular events and dialysis) and impact on the quality of life.

\section{METHODS}

\section{Study design}

This review protocol is in conformity with the Preferred Reporting Items for Systematic Review and Meta-Analysis Protocols (PRISMA-P) and will be reported in accordance with the PRISMA statement. ${ }^{14}$

\section{Protocol registration}

As recommended by the PRISMA-P guidelines, ${ }^{14}$ this review protocol was registered with PROSPERO International Prospective Register of Systematic Reviews in February 2018: registration number CRD42018088973. 


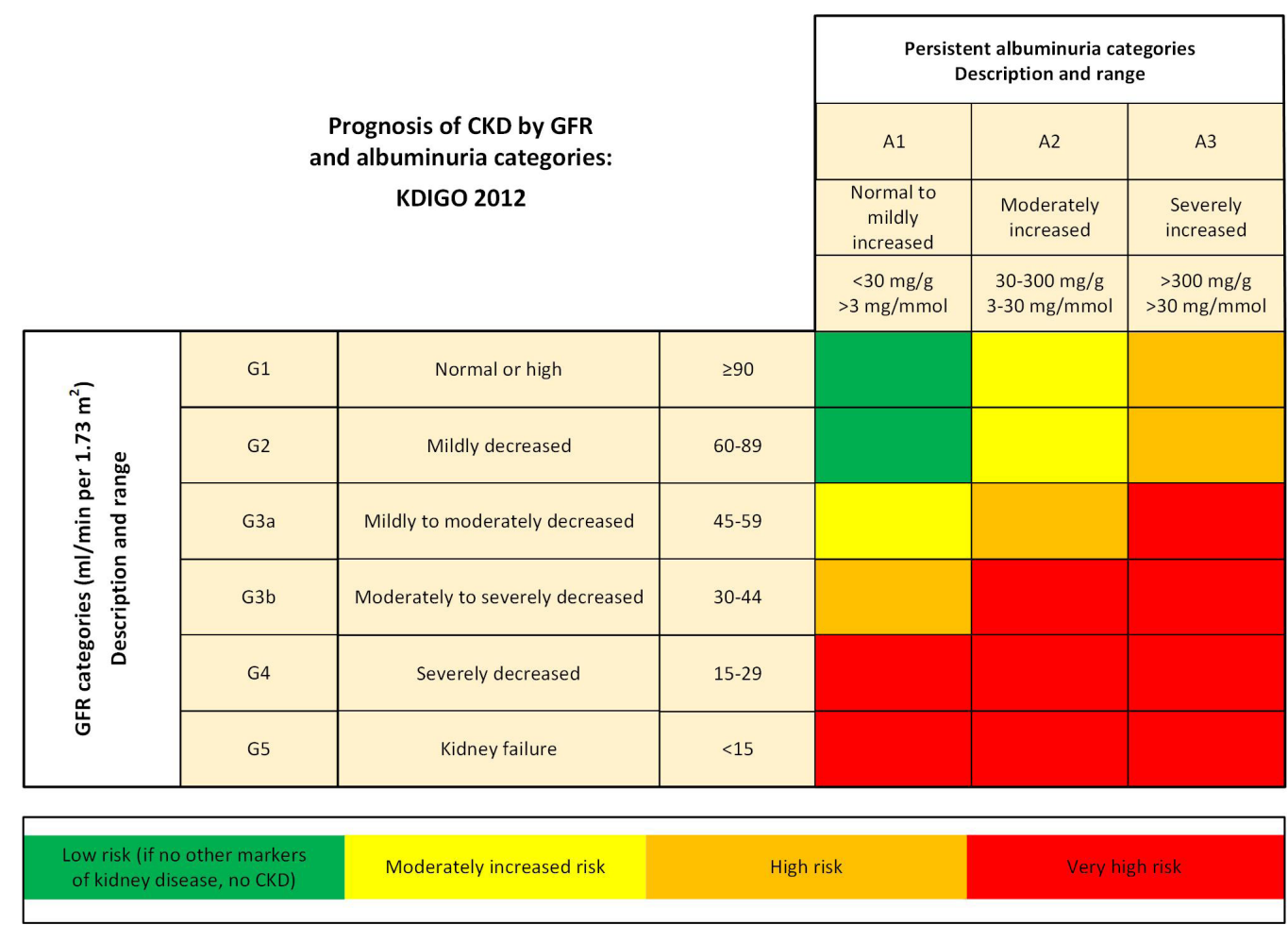

Figure 2 Stages of CKD. Reprinted with permission from KDIGO 2012 Clinical Practice Guidelines for the Evaluation and Management of Chronic Kidney Disease. ${ }^{32}$ GFR, glomerular filtration rate; KDIGO, Kidney Disease: Improving Global Outcomes.

\section{Inclusion criteria}

Studies of adult patients with CKD across the spectrum of CKD (as defined by international practice guidelines ${ }^{15}$ ) (figure 2) or kidney transplant recipients will be eligible for inclusion. No publication year limit will be applied. Published and peer-reviewed randomised controlled trials, observational studies including prospective and retrospective cohort studies (with and without control groups) will be included. In addition, relevant grey literature will be included. Reference lists of systematic reviews will be hand-searched; English and non-English language publications will be reviewed (including but not limited to Turkish, Arabic and Persian languages where a translator is available).

\section{Geography and seasonal trend}

As the Ramadan fast follows a lunar calendar and extends from sunrise to sunset, we will capture all studies across any climate zone to allow analysis stratified by seasonal and by climate variations (figure 1 ).

\section{Exclusion criteria}

Failure of texts to meet any of the above eligibility criteria will result in exclusion from the study. Studies involving patients receiving dialysis will also be excluded. Additionally, the following types of studies will be excluded due to the low strength of evidence: case reports, non-peer-reviewed publications, editorials, commentaries and opinion pieces.

\section{Search strategy}

We will use the services of a medical librarian with expertise in systematic reviews to do a comprehensive literature search using a combination of controlled vocabulary terms from the following platforms: Ovid and EBSCOhost. Electronic databases include MEDLINE, Embase, Global Health, CINAHL, Scopus, Web of Science and Google Scholar (online supplementary tables S2 and S3). Authors will be contacted for additional information such as missing outcome data when relevant. Health professionals and colleagues may be consulted in order to identify additional grey literature not identified through the literature search. Key search terms include 'Ramadan', 'fasting' and 'CKD' among others (online supplementary table S3). Additionally, reference lists of identified texts will be searched manually to identify other relevant texts, which will then be screened and selected, based on the described method.

\section{Data management}

Literature search results will be imported into EndNote X7. Databases in Microsoft Excel will be created to record results from each stage of the selection process. A data extraction database will also be developed in Microsoft Excel.

\section{Selection process}

Reviewers will follow the PRISMA recommended study flowchart in the selection process (figure 3).${ }^{14}$ A relevance form will be developed outlining the eligibility criteria and will be used to guide the abstract screening process 


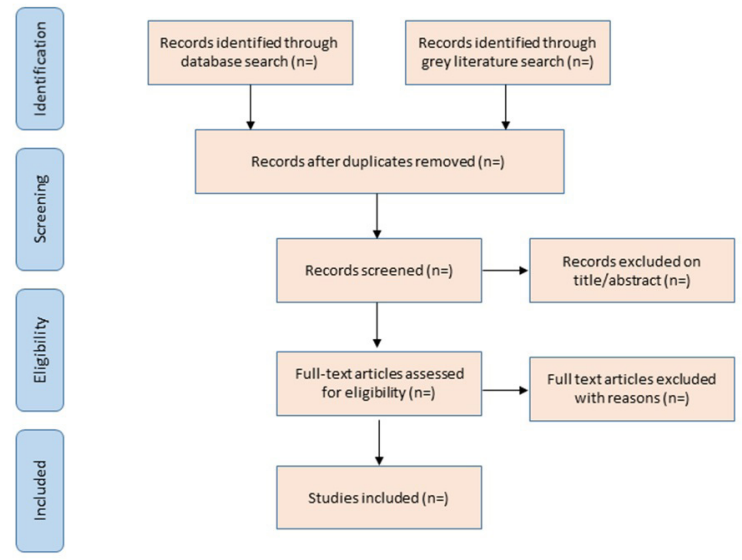

Figure 3 Flowchart-study selection.

and be a formal tool for recording results from the fulltext review process. Two reviewers will independently perform title and abstract screenings. Articles that do not meet the inclusion criteria at this stage will be excluded. Following initial title and abstract screening, full-text articles will be retrieved and assessed for eligibility for the study. Two reviewers will (a) perform a calibration exercise on five full-text articles and revise the relevance form as necessary, then (b) independently review all full-text articles, whereas a third reviewer will review any discrepancies. For any excluded study, one of the predefined exclusion criteria will be recorded in the database. Where a translator is available, non-English titles/abstracts/fulltext articles will be translated.

\section{Data collection process and data items}

One reviewer will extract data using the data extraction database and a second will independently check the data. Disagreements will be resolved by a third reviewer. Items recorded in the database will include: study characteristics (country, design, sample size and season), participants (patient demographics, kidney function (serum creatinine and/or estimated glomerular filtration rate), CKD status, comorbidities and medications used), exposure group information (fasting duration, control group details and co-interventions) and outcomes (timing of outcome, results-for all outcomes specified) (online supplementary table S4).

\section{Outcomes and prioritisation}

The primary outcome of interest is change in kidney function (defined as the change in serum creatinine level (SCr) or estimated glomerular filtration rate (eGFR)). The generally accepted standard to indicate the presence of CKD is a persistent eGFR of $<60 \mathrm{~mL} / \mathrm{min} / 1.73 \mathrm{~m}^{2}$; however, SCr alone is an acceptable alternative for the purposes of diagnosis or monitoring though concordance is imperfect. ${ }^{1101116}$

Secondary outcomes include (a) changes in risk factors such as change in proteinuria, blood pressure, blood glucose concentrations and weight/body mass index

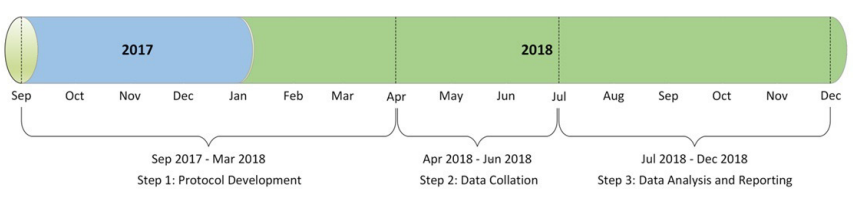

Figure 4 Project timeline.

(BMI), (b) electrolytes disorders such as hyperkalaemia, (c) episodes of AKI, (d) all-cause hospitalisation, (e) dialysis, (f) cardiovascular events (myocardial infarction and stroke) and (g) impact on the quality of life. Identifying a broad range of outcomes will allow for a more comprehensive synthesis of the evidence regarding the effects of Ramadan fasting on CKD/kidney transplantation.

\section{Timeline}

Data collection will take place from April to June 2018 with data analyses and reporting from July to December 2018 (figure 4).

\section{Risk of bias in individual studies}

Two reviewers will independently assess all included studies for methodological validity using criteria from the Downs and Black checklist (which is appropriate for both randomised and non-randomised studies). ${ }^{17}$ Evaluating criteria include study design, approach to recruitment, sample size calculation and reporting of missing primary outcome measurements. We considered other criteria such as the Newcastle-Ottawa Scale, but its criterion validity as well as the inter-rater and intrarater reliabilities are less well defined compared with the Downs and Black method. ${ }^{18}$

Source of funding will also be extracted given its potential to introduce bias. ${ }^{19}$ Prior to formally assessing the risk of bias, a calibration exercise will be performed on five included studies and the risk of bias form will be revised as necessary. Discrepancies will be resolved by a third reviewer. If possible, we will assess for the presence of publication bias. ${ }^{20}$

\section{Statistical analysis and data synthesis}

The mean difference or the risk ratio will be used to summarise continuous and dichotomous outcomes. If enough data are identified allowing for meta-analysis, results will be pooled using a random-effects model ${ }^{21}$ where clinical and methodological heterogeneity is reasonable. Statistical heterogeneity will be quantified using the $I^{2}$ statistic. ${ }^{22}{ }^{23}$ If possible, we will examine the following subgroups (CKD stage, latitude, season and risk of bias items) for evidence of effect modification on the association between fasting and each of the primary outcomes. Analyses will be performed using Stata/MP, V.15.1.

\section{Patient and public involvement}

Patients and the public were not involved at this stage of the project. 


\section{DISCUSSION}

This review aims to summarise the effects of Ramadan fasting on renal function and related outcomes in patients with CKD and/or renal transplant recipients. The literature on this topic is lacking with no guidelines to assist clinicians in advising their patients about the risks of Ramadan fasting on their kidney function. One previously published narrative review suggested the absence of any adverse associations of Ramadan fasting on the heart, lung, liver, kidney, eyes, haematological profile, endocrine and neuropsychiatric functions among healthy participants, ${ }^{24}$ whereas other studies suggest benefits in weight reduction and improvements with blood pressure control. ${ }^{25}$ However, these studies typically excluded patients with renal impairment.

The few studies that have specifically included patients with renal impairment have significant limitations affecting their interpretation and are, at times, contradictory. A small systematic review of patients with CKD demonstrated similar benefits in weight reduction and improvements with blood pressure control as in the general population. ${ }^{1}$ Hendawy suggests that there is no deleterious effect on renal function among patients who underwent kidney transplant when fasting for $\leq 12$ hours, but acknowledges this may not be generalisable to fasting for a more extended duration. ${ }^{13}$ A review by Bragazzi concludes that there is insufficient evidence to indicate Ramadan fasting is injurious to patients with $\mathrm{CKD} .{ }^{26}$ Conversely, other literature suggests that Ramadan fasting could be harmful to patients with pre-existing cardiovascular disease (common in patients with CKD) as it is associated with a high risk of acute cardiovascular events including heart attacks and strokes. ${ }^{12}{ }^{13}$ Data on changes in kidney function, as demonstrated by increased serum creatinine and/or decreased eGFR, are more limited. ${ }^{1}$ However, this is vital information in CKD management as there is a particular concern that medications widely used in the management of CKD (inhibitors of the reninangiotensin-aldosterone system) may amplify adverse changes in kidney function in the fasting state. This underscores the need for a more comprehensive systematic review of the literature.

The findings generated by this review have the potential to inform practice in the management of patients with CKD aiming to observe the Ramadan fast across the world. This study may lead to new insights into the associations of fasting with kidney function loss, changes in risk factors, adverse health outcomes and quality of life. It may also serve to identify gaps in the current literature and point to future directions for research. To our knowledge, this is the first attempt to systematically review and synthesise literature on the effects of Ramadan fasting across the spectrum of CKD and describe the association with a wide range of important clinical outcomes. Overall, this systematic review is expected to be relevant to a number of health professionals and patients.

Anticipated limitations include a small number of publications on this topic, publication bias and variety of languages of publications. In order to address the limited number of citations and potential publication bias, this review will be rigorous in that references of identified systematic reviews on the topic will be manually searched and assessed for inclusion in addition to the grey literature $^{27}$ and will not be limited by country or language. We will use translation services to translate relevant publications as needed.

\section{Ethics and dissemination}

Ethics is not required as this is a systematic review of previously published research. This review will provide comprehensive information on the effect of Ramadan fasting on renal function and related outcomes. To communicate our research findings, we plan to undertake a multifaceted approach for dissemination. ${ }^{28}{ }^{29}$ First, we plan to publish the results in an open access, peer-reviewed journal for broader exposure to the research and clinical community. Second, results will be presented at relevant conferences/ scientific meetings. Third, as the research has the potential to inform clinical practice, we will engage the relevant stakeholders and study groups such as the Ramadan Study Group, ${ }^{30}$ Diabetes and Ramadan International Alliance, ${ }^{31}$ and experts on kidney care (Kidney Disease: Improving Global Outcomes (KDIGO), ${ }^{32}$ International Society of Nephrology) ${ }^{33}$ to ensure the application of study findings into practice guidelines for practitioners and policy-makers. Finally, lay summaries will be provided to patients and their care providers.

Acknowledgements Authors would like to thank Thane Chambers for her expertise in the development of the search strategy.

Contributors RPP, AKB, SH: conceived the original idea for this study. AKB, JK, MAO, AL and RPP: wrote the first draft of the manuscript. MEO, NW, UQ and SS: contributed to the study protocol development, reviewed the manuscript for intellectual content and approved the final submitted version of the manuscript.

Funding This work is funded by a grant from Northern Alberta Renal Program.

Map disclaimer The depiction of boundaries on the map(s) in this article do not imply the expression of any opinion whatsoever on the part of BMJ (or any member of its group) concerning the legal status of any country, territory, jurisdiction or area or of its authorities. The map(s) are provided without any warranty of any kind, either express or implied.

Competing interests None declared.

Ethics approval Approval by research ethics board is not required since the review will only include published and publicly accessible data.

Provenance and peer review Not commissioned; externally peer reviewed.

Open access This is an open access article distributed in accordance with the Creative Commons Attribution Non Commercial (CC BY-NC 4.0) license, which permits others to distribute, remix, adapt, build upon this work non-commercially, and license their derivative works on different terms, provided the original work is properly cited, appropriate credit is given, any changes made indicated, and the use is non-commercial. See: http://creativecommons.org/licenses/by-nc/4.0/.

\section{REFERENCES}

1. Bragazzi NL. Ramadan fasting and chronic kidney disease: does estimated glomerular filtration rate change after and before Ramadan? Insights from a mini meta-analysis. Int J Nephrol Renovasc Dis 2015;8:53-7.

2. Finder I. Prayer Times Today. 2018 https://www.islamicfinder.org/ (Accessed 13 Mar 2018). 
3. Kottek M, Grieser J, Beck C, et al. World Map of the KöppenGeiger climate classification updated. Meteorologische Zeitschrift 2006;15:259-63.

4. Pew Research Center. Mapping the global Muslim popultion: a report on the size and distribution of the world's Muslim population: The World Bank, 2009.

5. Statistics Canada. 2011 National Household Survey: Data tables. 2011. http://www12.statcan.gc.ca/nhs-enm/2011/dp-pd/dt-td/indexeng.cfm (Accessed Mar 13, 2018).

6. Haddad YY, Quadri J. Islam. 2011. http://www.thecanadianencyc lopedia.ca/en/article/islam/ (Accessed 13 Mar 2018).

7. Al Wakeel J, Mitwalli AH, Alsuwaida A, et al. Recommendations for fasting in Ramadan for patients on peritoneal dialysis. Perit Dial Int 2013;33:86-91.

8. Pathy R, Mills KE, Gazeley S, et al. Health is a spiritual thing: perspectives of health care professionals and female Somali and Bangladeshi women on the health impacts of fasting during Ramadan. Ethn Health 2011;16:43-56.

9. Koushali AN, Hajiamini Z, Ebadi A, et al. Effect of Ramadan fasting on emotional reactions in nurses. Iran J Nurs Midwifery Res 2013;18:232.

10. Bernieh B, Al Hakim MR, Boobes Y, et al. Fasting Ramadan in chronic kidney disease patients: clinical and biochemical effects. Saudi J Kidney Dis Transpl 2010;21:898-902.

11. El-Wakil HS, Desoky I, Lotfy N, et al. Fasting the month of Ramadan by Muslims: could it be injurious to their kidneys? Saudi J Kidney Dis Transpl 2007;18:349-54.

12. NasrAllah MM, Osman NA. Fasting during the month of Ramadan among patients with chronic kidney disease: renal and cardiovascular outcomes. Clin Kidney J 2014;7:348-53.

13. Hendawy A. Effect of ramadan fasting on renal physiology. Urol Nephrol Open Access J 2014;1.

14. Moher D, Shamseer L, Clarke M, et al. Preferred reporting items for systematic review and meta-analysis protocols (PRISMA-P) 2015 statement. Syst Rev 2015;4:1.

15. Kidney Disease: Improving Global Outcomes (KDIGO) CKD-MBD Update Work Group. KDIGO 2017 Clinical Practice Guideline Update for the Diagnosis, Evaluation, Prevention, and Treatment of Chronic Kidney Disease-Mineral and Bone Disorder (CKD-MBD). Kidney Int Suppl 2017;7:1-59.

16. Bello AK LA, Tonelli M, Okpechi IG, et al. Global kidney health atlas: a report by the International Society of Nephrology on the current state of organization and structure for kidney care across the globe. Brussels, Belgium: International Society of Nephrology, 2017.

17. Downs $\mathrm{SH}$, Black $\mathrm{N}$. The feasibility of creating a checklist for the assessment of the methodological quality both of randomised and non-randomised studies of health care interventions. J Epidemiol Community Health 1998;52:377-84.

18. Hartling L, Milne A, Hamm MP, et al. Testing the Newcastle Ottawa Scale showed low reliability between individual reviewers. J Clin Epidemiol 2013;66:982-93.

19. Cho MK, Bero LA. The quality of drug studies published in symposium proceedings. Ann Intern Med 1996;124:485-9.

20. Song F, Eastwood AJ, Gilbody S, et al. Publication and related biases. Health Technol Assess 2000;4:1-115.

21. Cornell JE, Mulrow CD, Localio R, et al. Random-effects metaanalysis of inconsistent effects: a time for change. Ann Intern Med 2014;160:267-270-70.

22. Higgins JP, Thompson SG. Quantifying heterogeneity in a metaanalysis. Stat Med 2002;21:1539-58.

23. Higgins JP, Thompson SG, Deeks JJ, et al. Measuring inconsistency in meta-analyses. BMJ 2003;327:557-60.

24. Azizi F. Islamic fasting and health. Ann Nutr Metab 2010;56:273-82.

25. Toda M, Morimoto K. [Effects of Ramadan fasting on the health of Muslims]. Nihon Eiseigaku Zasshi 2000;54:592-6.

26. Bragazzi NL. Ramadan fasting and chronic kidney disease: $A$ systematic review. J Res Med Sci 2014;19:665-76.

27. McAuley L, Pham Ba', Tugwell P, et al. Does the inclusion of grey literature influence estimates of intervention effectiveness reported in meta-analyses? The Lancet 2000;356:1228-31.

28. Bero LA, Grilli R, Grimshaw JM, et al. Closing the gap between research and practice: an overview of systematic reviews of interventions to promote the implementation of research findings. The Cochrane Effective Practice and Organization of Care Review Group. BMJ 1998;317:465-8.

29. Kerner JF, Hall KL. Research dissemination and diffusion: Translation within science and society. Res Soc Work Pract 2009;19:519-30.

30. Masood SN, Alvi SFD, Ahmedani MY, et al. Comparison of Ramadanspecific education level in patients with diabetes seen at a Primary and a Tertiary care center of Karachi-Pakistan. Diabetes Metab Syndr 2014;8:225-9.

31. Nematy M, Mehdizadeh A. Introducing the practical guideline for Diabetes and Ramadan, developed by international Diabetes federation in collaboration with Diabetes and Ramadan International Alliance, 2016. J Fasting Health 2016;4:95-6.

32. KDIGO. 2012 Clinical Practice Guideline for the Evaluation and Management of Chronic Kidney Disease. Kidney Int Supp 2013;3:19-62.

33. Levin A, Eckardt K-U, Tonelli M. The Global Kidney Health Summit Outputs: details to guide the nephrology community along the road to global kidney health. Kidney Int Suppl 2017;7:61-2. 